\section{ALGUNOS ASPECTOS TECNICOS ESTRUCTURALES QUE CONDICIONAN LA REALIZACIÓN DE UNA OBRA DE REMODELACION}

\author{
Enrique González Valle \\ Ingeniero de Caminos \\ Subdirector General de INTEMAC
}

\section{RESUMEN:}

El autor esboza una sistemática del estudio previo al que debe ajustanse una obra de remodelación que afecte a los elementos estructurales de una construcción. pasando revista a los problemas que suelen plantearse y a los medios auxiliares que pueden aplicarse para eliminar las incertidumbres que condicionarian la seguridad y durabilidad de las elementos originales afectados por la obra.

\section{Introducción}

El planteamiento de una obra de remodelación debe partir de una base fundamental: el conocimiento exacto de la situación de construcción de la obra. Tal conocimiento es imprescindible cuando entre los elementos afectados están los inte grantes de la estructura del ediricio, ya que, in trínsecamente, muchos son los factores que pueden afectar a las condiciones de seguridad de los mismos.

El caso concreto de los estadios merece un análisis particularizado, ya que las obras de remodelam ción suponen, en múltiples casos, cambio de las condiciones de utilización de elementos, fundamentalmente estructurales de hormigón armado, que además involucran la responsabilidad de los autores del proyecto de remodelación.

Por ello, nos ha parecido oportuno abordar este tema que no điene más objeto que plantear un procedimiento racional de valoración, por una parte, de las condiciones de resistencia y durabilidad de la solución original $y$, por otra, de la situación alterada por la remodelación, para, en base a tales valoraciones técnicas, establecer los condicionantes a que la obra deba ajustarse, definir las obras del reluerzo a realizar y, en su caso, proceder a un análisis comparativo, desde el punto de vista económico, con soluciones de obra nueva.

Lo que sigue debe entenderse como un esbozo de sistemática, subjetivamente estabecida, a la que debe ajustarse cualquier reforma o modificación de las condiciones de uso en elementos estructurales. Si hacemos referencia particular a los estadios no es sino porque, en general, tales construcciones están integradas por elementos fundamentalmente estructurales.

Para una mejor ordenación, dividimos el procedim miento que exponemos en cuatro fases fundamentales, en cada una de las cuales planteamos los aspectos técnicos que las condicionan y sugerimos posibles vias de estudio. Tales fases son, en esencia:

- Análisis de la normativa existente en la época de realización del proyecto y otros aspectos técnicos que enmarcan los antecedentes.

- Análisis de la documentación del proyecto caso de existir y realización de contrastes in situ para establecer su flabilidad.

- Análisis de las caracteristicas resistentes de los materiales empleados y sus condiciones de durabilidad.

- Evaluación de los niveles de seguridad que presenta la construcción sin reformar, a la luz de la normativa vigente, y consideración de los condicionantes que se establecen para la re modelación.

\section{Análisis de la normativa vigente en las épocas de realización del proyecto y otros aspectos básicos que enmarcan los antecedentes}

La normativa que reglamenta, tanto las acciones a considerar, como el proyecto de elementos de hormigón armado y pretensado, ha evolucionado, ultimamente, de forma extraordinaria. Normas que estuvieron en vigor durante más de veinticinco 
anos, sin cambios fundamentales, se han visto modiflcadas por varias ediciones que incorporan nuevos criterios, tanto en el establecimiento de las hipótesis de cálculo, como en el comporta miento nominal de las secciones. Refiriéndonos al caso de España, hasta 1968 han sido aplicados criterios de cálculo clásico y, en teoria, ajustándo se a tales principios fueron calculadas múltiples estructuras pertenecientes a nuestros estadios hoy remodelados. Desde 1968 hasta nuestros dias, han aparecido tres ediciones de la norma que reglam menta las construcciones de hormigón amado (EH-68, EH-73 y EH-80) las cuales han supuesto cambios fundamentales, tanto en los criterios básicos de cálculo de secciones. como en las especificaciones relativas a la disposición de detalle de las armaduras.

El caso español no es único y se encuentra den tro del contexto de las reglamentaciones ajustadas al CEB (Comité Euro-internacional del Hormigón), pudiendo citarse casos parecidos en casl todos los paises europeos y en sus diferentes áreas de influencia. Igualmente podriamos decir en relación a la nomativa americana, fundamentalmente estam blecida en base a la norma ACI-318, y a su área de influencia.

Paralelamente, los criterios de cálculo de esfuerzos en estructuras han evolucionado hundamental mente, en base a investigaciones modernas reall. zadas sobre el comportamiento de entramados, variandose muchos de los planteamientos (podriamos decir que clásicos o lineales) que tradicional mente venian siendo empleados, los cuales tienden a ser sustituidos por otros criterios o hipóle sis de comportamiento más ajustados a la reallm dad contrastada por la investigación o por estum dios a nivel teórico que, en estos momentos, son viables por la utilización de computadoras de maw yor potencia.

En muchos casos, nuestras estructuras fueron realizadas con materiales que han evolucionado y que no existen actualmente en el mercado, por lo que resulta dificil establecer las condiciones que a los mismos les eran exigidas, en momentos en que, además, era escasa la normalización al respecto.

Ante tales aspectos y de cara a una obra de remodelación, es preciso conocer en detalle cuáles fueron los condicionantes, normativos o de mercado, a que se ajustó (o más propiamente, debió ajustarse) nuestra construcción, dado que ello es básico para poder establecer con llabilidad el anám lisis de una estructura cuyas condiciones de uso, o de comportamiento, van a ser modificadas.
El conocimiento de tal normativa, la cual generalmente es accesible tras búsqueda bibliográfica, nos permite ponernos en la posición del proyeclista ante la obra, gozando además de una mayor capacidad de interpretación de la misma, dado que, en muchos casos, las diferencias que encontremos con las especificaciones actuales serán puntos en los cuales deberemos centramos para efectuar un análisis de detalle, recurriendo a la bibliografia especializada que haya analizado los antecedentes de la modificación.

En lo que respecta a las características de los materiales empleados, kambién se requerirá una recoplación y análisis de la documentación técnica y demás antecedentes que existan sobre el empleo de tales productos (caracteristicas de cementos y amaduras empleadas, fundamentalmente) asi como su valoración en el tiempo en base a la experiencia recogida en su utilización.

Por ultimo, pueden existir otros antecedentes, tales como tecnología y procedimientos de construcción de uso común, cuyo análisis puede resultar adecuado para la valoración de la situación general. También convendrá efectuar estudios es. pecificos ante anomallas locales detectadas en otras fases.

\section{Análisis de la documentación de proyecto, caso de existir y realización de contrastes in situ para establecer su fiabilidad}

Como fase siguiente a la que podriamos calificar de puesta en situación ideal de proyecto en tiempo y situación, establecemos como necesario un análisis de la documentación de proyecto, lo que en general conlleva múliples y a veces insalvables dificultades.

En general, nos encontramos con dos tipos de problemas, que vienen originados por causas ligadas a la reglamentación técnica y a la normativa legal de los diferentes paises. En primer lugar, se ha entendido por muchos técnicos que el plazo de responsabilidad legal, decenal en su caso, es el que marca la responsabilidad de guardar en sus archivos la documentación relativa a las obras. Con independencia de ello, el propietario ha efectuado en ocasiones pequeñas reformas, gran número de las cuales no suelen encontrarse documentadas por no tener conciencia clara de que asi es impuesto por la normativa legal, que le hace responsable a tales efectos. 


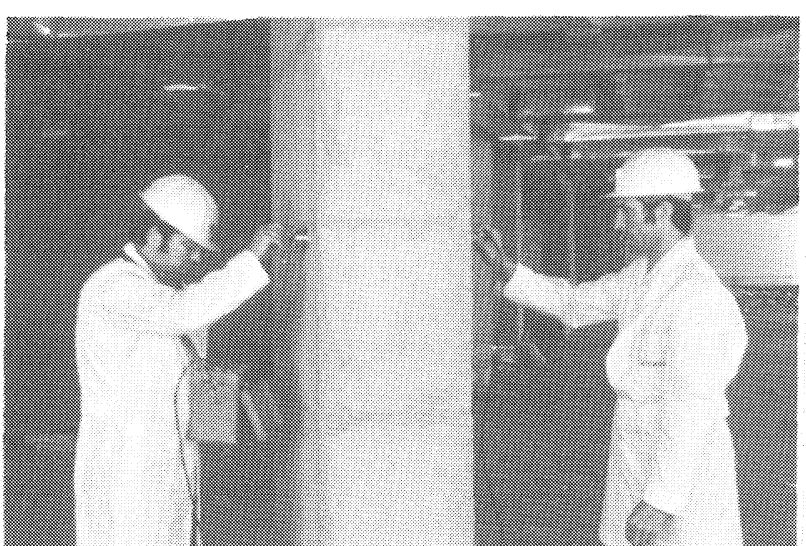

Medidas realizadas mediante ultrasonidos.

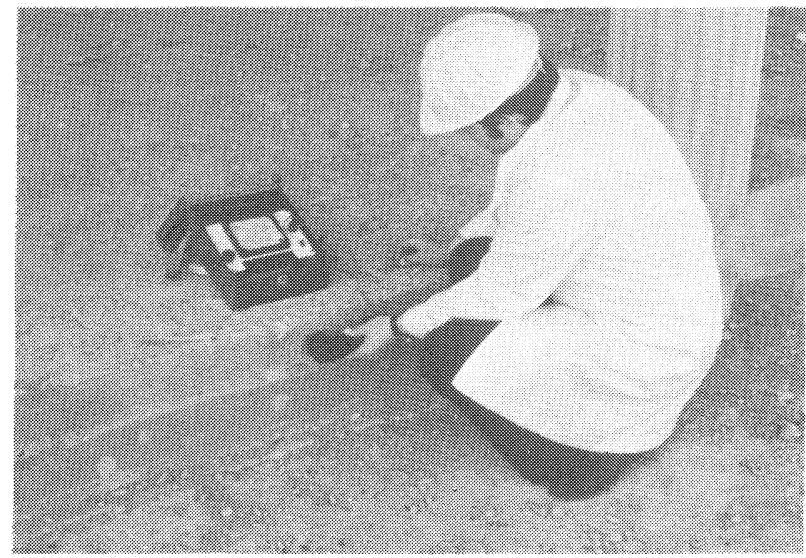

Sonda magnética de detección de armaduras.

En consecuencia, son múltiples los casos en los cuales, o bien no es posible encontrar la documentación acreditativa de la situación de cons trucción, o bien la misma carece de la fiablidad suficiente para reflejar tal situación.

Evidentemente, la base de partida de una obra de remodelación debe ser el conocimiento detallado de la obra original. Si no está documentada fiablemente, cosa que es habitual, es necesario re currir a toma de datos directamente in situ, 10 cual, aunque en un estadio se facilita por la existencla de múltiples elementos análogos, plantea todas las dificultades que conlleva el reconocimiento de una obra de hormigón armado en la que, con independencia de las dimensiones, es necesario conocer el trazado de las armaduras.

La utilización de sondas magnéticas de armadu ras, complementadas con rozas, puede constituir un elemento auxiliar valioso para el reconocimiento de estructuras en tales situaciones, pudiendo incluso recurrirse a procedimientos más sofisticados, tales como la radiografia, si determinados puntos se consideran esenciales para analizar el comportamiento estructural.
El reconocimiento de toma de datos y contraste de la documentación, debe complementarse con una evaluación general de las condiciones resistentes y de durabilidad, mediante una inspección al efecto. Tambien en estos casos son aplicables los medios auxiliares de inspección antes citados (sondas magnéticas, ultrasonidos, etc.) para definir preliminarmente la trascendencla de los daños que encontramos (anchos y profundidades de fisuras, ausencia de determinadas armaduras, etc.) y poder centrar el estudio de seguridad en aquellos puntos que la experiencia de utilización del elemento ha mostrado como sensibles. En principio, el reconocimiento o investigación de los danos no tiene porqué hacer presumir unas condiciones anómalas del comportamiento resistente, ya que existen muchos de ellos que no afectan significativamente a los aspectos resistentes fundamentales; pero cuando menos, siempre contribuirá este reconocimiento a mejorar las condiciones de durabilidad o a adoptar disposiciones de construcción que permitan corregir los defectos de tales puntos dañados.

\section{Análisis de las características resistentes de los materiales empleados $y$ de sus condiciones de durabilidad}

El siguiente paso que daremos en un estudio de remodelación es la investigación de los materiam les componentes de la estructura, para evaluar sus caracteristicas resistentes y sus condiciones de durabilidad.

En general, las obras de hormigón armado presentan, por una parte, incrementos de resistencia apreciables con la edad, que son función del tipo de cemento, dosificación y áridos empleados. Pero, por otra parte, la tecnologia de construcción empleada en tiempos pasados adolecía a menudo de falta de precauciones e incluso de medios, para garantizar condiciones de durabilidad alta. Podriamos añadir a 10 anterior el que, generalmente, muchas de estas construcciones presentan su estructura a la intemperie $y$, en consecuencia, expuesta a la agresión del ambiente.

Situaciones de seguridad precaria, nominalmente o de proyecto, pueden haber sido subsanadas por el incremento de resistencias del hormigón con el tiempo, fundamentalmente en elementos comprimidos. Pero a la vez, las coqueras, falta de compacidad, calidad del hormigón para preservar de la corrosión a las armaduras, etc. pueden haber ori- 
ginado bajas de seguridad en los elementos en llexion, hasta plantear siluaciones precarlas. Todo ello unido a que, normalmente, no es usual que existan esquemas de mantenimiento en estructum ras al igual que se plantea en otros componentes como cubiertas, instalaciones, etc.

Ante esto se hace necesario evaluar tanto las caracteristicas resistentes como las condiciones de durabilidad, mediante investigación de las calidades de los materiales alterados por el paso de los años; evaluación que puede condicionar seriamente la remodelación, exigiendo soluciones de reparación o refuerzo que la hagan inviable desde un punto de vista económico. Asi ha podido constatarse en algunas de las realizaciones españolas.

Las técnicas empleadas para esta investigación consisten generalmente en la combinación de procedimientos de ensayo destructivo y no destructivo. La aplicación del esclerómetro y ultrasonidos, correlacionada con resultados de ensayos a comm presión sobre lestigos, se ha mostrado un buen procedimiento, ágil y fiable, para la investigación de la calidad del hormigón. Complementariamente, la utilización generalizada de procedimientos ultrasónicos, permite detectar defectos internos en el hormigón y delimitar el alcance de los defectos externos.

A fin de dictaminar sobre las condiciones de durabilidad de la estructura resulta muy conveniente sancionar fécnicamente la calidad del hormigón, en 10 que respecta a su contenido de cemento y granulometria. Sus resultados, en algunos casos, pueden hacer aconsejable tratamientos superficiales de durabilidad que permitan garantizar una vida media a la estructura remodelada comparable con la de una de nueva planta.

Por último, la investigación a que hacemos refe rencia debe, asimismo, afectar al estado y calidad

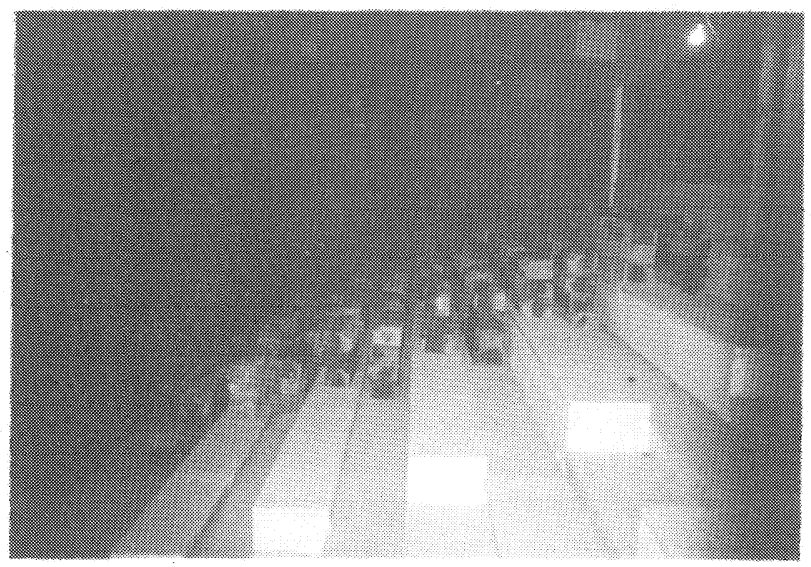

Prueba de carga en un graderio. del acero, con el criterio de que las muestras se tomen de puntos en los que estructuralmente la capacidad resistente no se vea afectada (o en su caso pueda ser repuesta) y de localizaciones significativas para poder emitir un juicio global de las condiciones de durabllidad de las armaduras.

El nivel de la investigación que realicemos, unido a la fiablidad de los datos tomados en la estructura, sobre su disposición de armaduras y dimensiones geométricas, puede condicionar el proceso de comprobación de los niveles de seguridad al canzados en las secciones básicas de la estructura, ya que un conocimiento exhaustivo de tales datos, en múltiples puntos, podria hacer válidos procedimientos de evaluación deterministas y con coeficientes de seguridad más ajustados, frente a los métodos semiprobabilistas y con adopción de los coeficientes de ponderación reglamentados.

Hemos omitido, deliberadamente, la utilización de las pruebas de carga como método de evaluación de las características de materiales y de la seguridad. Queremos llamar la atención sobre la gran ayuda que para el que tiene que dictaminar sobre la aceptación de una estructura, puede representar la realización de pruebas de carga. Pero entendemos que por si solas, siempre que no se apliquen según especifica $\mathrm{ACl}-318$ en su apartado sobre evaluación resistente de estructuras existentes, no tienen una validez determinante. Para ello su utilización debería generalizarse a múltiples elemen tos y hay que tener en cuenta que en algunos de ellos no seria posible reproducir las situaciones de ensayo que alli se preconizan, en lo referente a las cargas y medios de seguridad a disponer.

Las pruebas de carga así realizadas, es decir, en condiciones que superan las situaciones de servim cio significativamente, o las realizadas en condiciones de servicio como complemento a los análisis teóricos sobre siluaciones nominales (en las que cabe plantearse la duda sobre la aceptación técnica) tienen una gran validez, pero siempre que la realización se ajusta a procesos normalizados que permitan juzgar el comportamiento al menos endeformaciones, evolución de la fisuración y re cuperación alcanzada tras el ensayo.

\section{Evaluación de los niveles de seguridad que presenta la construcción. a la luz de la normativa vigente y condicionantes a la remodelación}

Las estructuras existentes sobre las cuales se pre tende actuar, pueden presentar la particularidad de 
que su seguridad nominal, o de proyecto, evalua da en base a la nomativa vigente, sea inferior a los niveles exigidos por tal normativa. Sin embar. go, tienen a su favor, en comparación con estructuras nuevas que deban ser proyectadas, la expe riencia de su uso en condiciones de servicio.

En base a tal principio, la siguiente lase que planteamos conduce al dilema de qué nivel de segurim dad, en situación nominal, debe exigirse a una estructura en la que, con independencia de las exigencias establecidas por las normas, se haya constatado un uso positivo en un tiempo dilatado. Evidentemente, la respuesta a tal dilema ha de ser que, constatada tal evidencia, pueden admitirse reducciones de seguridad nominal en aquellos aspectos de carácter no frágil en los cuales el daño se haga ostensible, previamente a la rotura, con sintomas diriamos que de aviso, materializados por ejemplo en la aparición de fisuras. Una simple comparación de los niveles de seguridad exigidos por diferentes normas, permitiría deducir que, al menos, una reducción del coelliciente de seguridad de un $10 \%$, podría ser justificado en cualm quier caso. Además, estando la estructura construida cabe la posibilidad de precisar, en forma determinista, las variables adoptadas en una comprobación semiprobabilista, lo que permite reducir los coeficientes de ponderación correspondientes. Ya antes hemos hecho referencia a otros aspectos al hablar de la investigación, sobre los procesos de comprobación de la seguridad por via determinista o semiprobabilista.

Bajo este prisma, deben sancionarse tanto los niveles de seguridad y el juicio sobre la aceptación de la obra original, como las especificaciones o criterios de cálculo ante la situación presumiblemente modificada por la remodelación.

En cualquier caso y planteada una situación que introduce, bien cambio en el uso, bien introducción de elementos que hacen variar el comportam miento estructural, parece obvia la necesidad de abordar esta lase para comprobar la adecuación de la obra al caso existente y normalizado en acciones, sus niveles de solicitación ante las nuevas condiciones de servicio, y los condicionantes estructurales a que deba ajustarse la remodelación, que, dicho sea de paso, también podria aliviar, cambiando el esquema estructural de los elemen tos existentes, su situación de seguridad.

\section{Epílogo}

El proceso que hemos expuesto debe ser desarrollado de una manera racional. Es preciso señalar

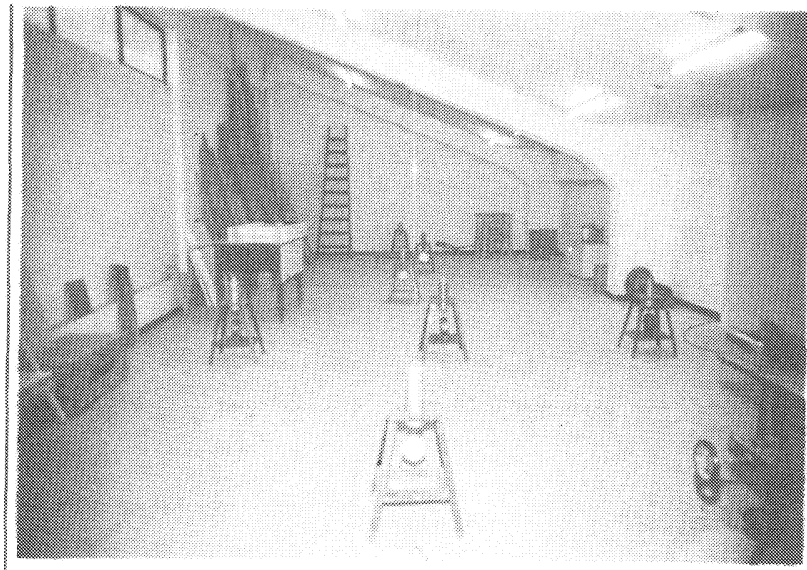

Disposición de fleximetros en una prueba de carga.

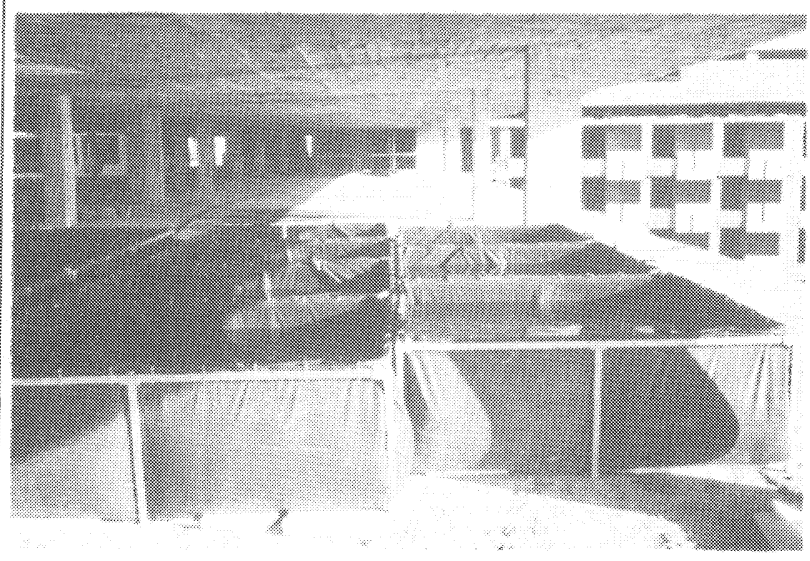

Forma de materializar la sobrecarga mediante balsas de agua.

que las exigencias de cumplimiento de una deter. minada planificación para la entrada en servicio, no permiten muchas veces disponer de los plazos necesarios para un estudio técnico de detalle de la situación, lo que impide desarrollar el proceso con la profundidad necesaria para garantizar la seguridad de la obra, llegándose a veces a solucio. nes de refuerzo no optimizadas tanto en plazo como en coste. Tales soluciones analizadas com parativamente con otras de obra nueva, pueden llevar incluso a decisiones de demolición.

También hay que dejar sentado, en el otro extre mo, que la raclonalldad del proceso impone contemplar a la construcción como tal y no como un banco de pruebas o laboratorio que permita investigar, en sus detalles, las tecnologias y materiales empleados en otras épocas, asi como el rendimiento estructural de las soluciones preconizadas por normativas anteriores. Para esas misiones están los centros de investigación, y en algunos cam sos las construcciones plloto. 
Aunque somos conscientes de que el fin último que debe pretenderse es la realización de la obra, ajustada a su planificación y a sus previsiones económicas, si queremos por último dejar consm tancia de un pensamiento acuñado entre la gente que ama la técnica: Es dificll no perder durante la construcción el tiempo que no fue dedicado a los estudios técnicos previos. Consideramos que este lema es muy aproplado para casos como el que nos ocupa, en los que la planificación olvida a menudo considerar los plazos necesarios para los estudios a que hemos hecho referencia.

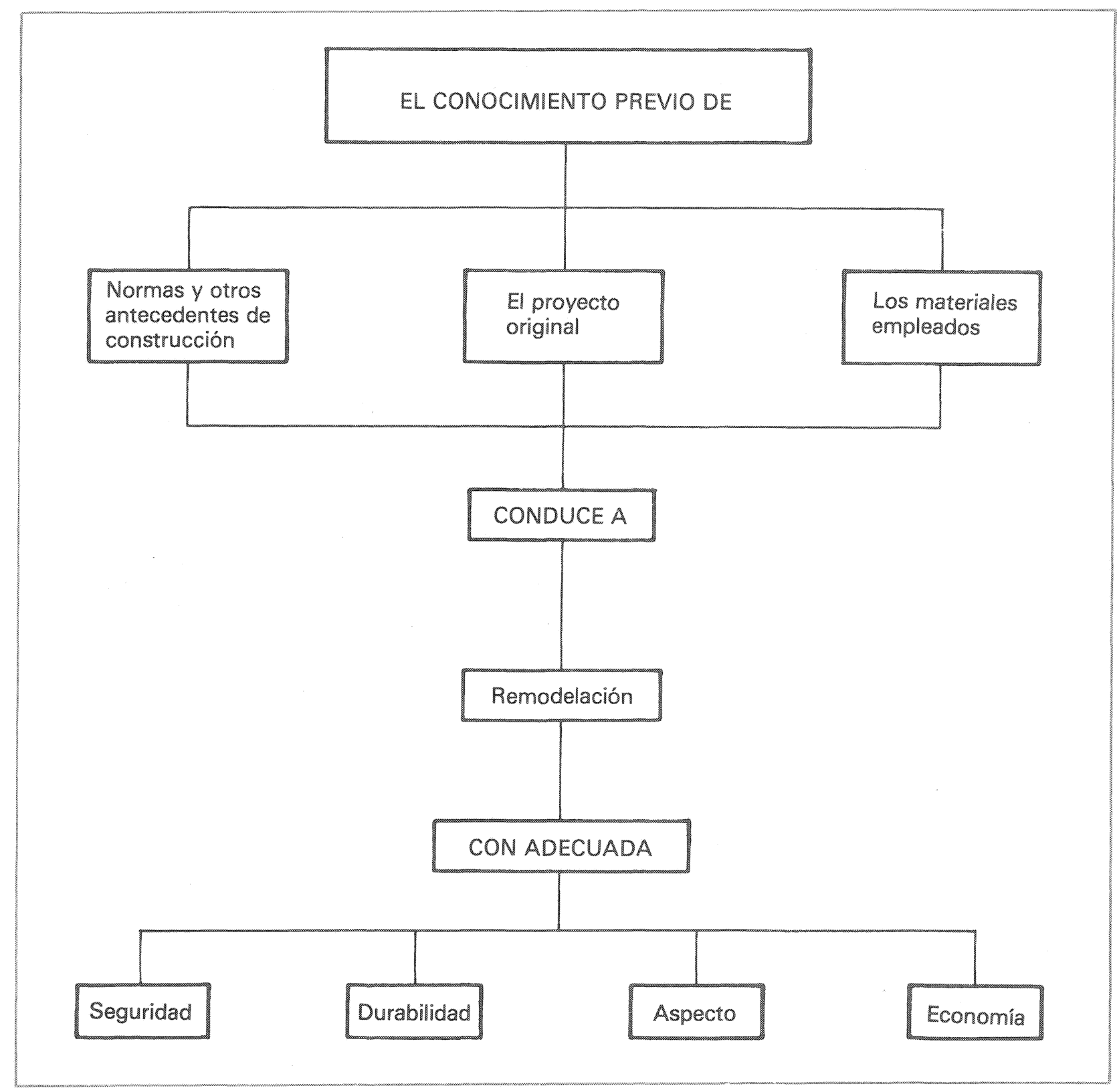

